JOURNAL OF

FUNCTION SPACES AND APPLICATIONS

Volume 4, Number 3 (2006), 305-327 (c) 2006, Scientific Horizon http://www.jfsa.net

\title{
$N$-harmonic extensions of weighted integrable distributions
}

\author{
Josefina Alvarez, Martha Guzmán-Partida \\ and \\ Salvador Pérez-Esteva* \\ (Communicated by Hans Triebel)
}

2000 Mathematics Subject Classification. 46F20, 46F05, 46F12.

Keywords and phrases. $S^{\prime}$-convolution, weighted distribution spaces, $n$ harmonic extensions of distributions.

\begin{abstract}
We obtain $n$-harmonic extensions to the Cartesian product of $n$ copies of the upper half-plane, of distributions in the weighted space $w_{1}^{2} \ldots w_{n}^{2} \mathcal{D}_{L^{1}}^{\prime}$, which is known to be the optimal space of tempered distributions that are $\mathcal{S}^{\prime}$ convolvable with a natural product domain version of the Poisson kernel.
\end{abstract}

\section{Introduction and notation}

The aim of this article is twofold. First, we solve the following boundary value problem: For each tempered distribution $T$ in an optimal class, we find a $n$-harmonic function $u\left(x_{1}, y_{1} ; \ldots ; x_{n}, y_{n}\right)$ on the product space $\mathbb{R}_{+}^{2} \times \ldots \times \mathbb{R}_{+}^{2}$ such that $u$ converges to $T$ in an appropriate sense when $y_{1}, \ldots, y_{n} \rightarrow 0^{+}$. Let us recall that a complex valued function

* Partially supported by PAPIIT-IN105606. 
$u\left(x_{1}, y_{1} ; \ldots ; x_{n}, y_{n}\right)$ is called $n$-harmonic if it is harmonic in each set of variables $\left(x_{j}, y_{j}\right)$ separately. Second, we characterize the class of $n$ harmonic functions on $\mathbb{R}_{+}^{2} \times \ldots \times \mathbb{R}_{+}^{2}$ that can be written as finite sums of products of one-dimensional Poisson integrals of appropriate distributions. The work of P. Sjögren in [14] plays a crucial role in the proof of such characterization.

Let us observe that to define the Poisson integral of a distribution, one must specify the rule of convolution and the class of distributions that will be considered. In fact, the Poisson kernel cannot be convolved with every tempered distribution if one expects to preserve the Fourier exchange formula. We consider here the $\mathcal{S}^{\prime}$-convolution developed by Y. Hirata and H. Ogata ([7]) and R. Shiraishi ([13]), to extend to appropriate pairs of tempered distributions the classical definition of convolution ([12]).

The results presented in this article concern a natural product domain version of the Poisson kernel, which is related to the notion of $n$-harmonic function. The optimal space of tempered distributions $\mathcal{S}^{\prime}$-convolvable with this version of the Poisson kernel is identified in [1] as a weighted space of distributions where the weight is a particular function $w$ with separable variables. The need to impose a weighted integrability condition in order to have a well defined Poisson integral, is already present in the case of functions. Indeed, as we show later, a measurable function $f$ has a well defined and everywhere finite Poisson integral if and only if $f$ is integrable with respect to the weight $w$. This observation extends to the product domain case the condition shown by T. M. Flett [6, p. 762] in the Euclidean case.

H. Bremermann studied in $[4$, p. 152] the boundary behavior of $n$ harmonic functions and obtained representation formulas by means of Poisson integrals. Our results extend the work of $\mathrm{H}$. Bremermann to a larger class of distributions that is optimal in the context of the $\mathcal{S}^{\prime}$-convolution.

This article is organized as follows: In Section 2 we collect definitions and results to be used later on. In Section 3 we obtain $n$-harmonic extensions to $\mathbb{R}_{+}^{2} \times \ldots \times \mathbb{R}_{+}^{2}$ of appropriate weighted integrable distributions. In Section 4 , we characterize a class of $n$-harmonic functions on $\mathbb{R}_{+}^{2} \times \ldots \times \mathbb{R}_{+}^{2}$ as finite sums of products of one-dimensional Poisson integrals. We must point out that this characterization still leaves open the problem of characterizing any $n$-harmonic function in terms of the convolution of appropriate distributions with the product domain Poisson kernel. We close our article with a Section 5 where we establish $\mathcal{S}^{\prime}$-convolvability results with Poisson like kernels, both in the Euclidean case and the product domain case. At the heart of this section is the observation that the $\mathcal{S}^{\prime}$-convolvability results rely more on having the appropriate estimates than on having exact formulas for the kernels under consideration. 
The notation used here is standard. The symbols $C_{0}^{\infty}, \mathcal{S}, C^{\infty}, L^{p}, \mathcal{D}^{\prime}$, $\mathcal{S}^{\prime}, \mathcal{E}^{\prime}$, etc., indicate the usual spaces of distributions or functions defined on $\mathbb{R}^{n}$, with complex values. With $L^{p}(\omega)$ we indicate those functions that are $p$-integrable with respect to the measure $\omega d x$ for an appropriate weight $\omega$. We denote $|\cdot|$ the Euclidean norm in $\mathbb{R}^{n}$, while $\|\cdot\|_{p}$ indicates the norm in the space $L^{p}$. When we work on a specific space other than $\mathbb{R}^{n}$, we will write $\mathcal{D}^{\prime}(\mathbb{R}), \mathcal{S}\left(\mathbb{R}^{2}\right),\|\cdot\|_{L^{p}(K)}$, etc., as appropriate. Partial derivatives will be denoted as $\partial^{\alpha}$, where $\alpha$ is a multi-index $\left(\alpha_{1}, \ldots, \alpha_{n}\right)$. If it is necessary to indicate on which variable we are taking the derivative, we will do so by attaching a sub-index. We will use the standard abbreviations $|\alpha|=\alpha_{1}+\ldots+\alpha_{n}, x^{\alpha}=x_{1}^{\alpha_{1}} \ldots x_{n}^{\alpha_{n}}$. For a function $g$, we will indicate with $\stackrel{\vee}{g}$ the function $x \rightarrow g(-x)$. Given a distribution $T$, we will denote with $\check{T}$ the distribution $\varphi \rightarrow(T, \stackrel{\vee}{\varphi})$, where $\varphi$ is an appropriate test function. The Fourier transform will be denoted as $\mathcal{F}$. A smooth function is a continuous function with continuous derivatives of all orders. The letter $C$ will indicate a positive constant, probably different at different occurrences. When it is important to indicate that the constant depends on certain parameters, we will do so by attaching sub-indexes to the constant.

Other notation will be introduced at the appropriate time.

\section{Preliminaries}

In order to introduce the notion of $\mathcal{S}^{\prime}$-convolution that we will extensively use, we shall first give a brief review of the spaces of functions and distributions related to this notion. All the notions and results can be found in [12] and [3].

The space of integrable distributions $\mathcal{D}_{L^{1}}^{\prime}$ is, by definition, the strong dual of the space $\dot{B}$ of smooth functions $\varphi: \mathbb{R}^{n} \rightarrow \mathbb{C}$ for which $\partial^{\alpha} \varphi \rightarrow 0$ as $|x| \rightarrow \infty$, for each multi-index $\alpha$. The space $\dot{B}$ is a closed subspace of the space $B$ consisting of those smooth functions $\varphi$ with the property that $\partial^{\alpha} \varphi$ is bounded for every multi-index $\alpha$, endowed with the topology of the uniform convergence on $\mathbb{R}^{n}$ of each derivative. The space $C_{0}^{\infty}$ of compactly supported smooth functions defined on $\mathbb{R}^{n}$, is dense in $\dot{B}$ but not in $B$. Thus, the space $\mathcal{D}_{L^{1}}^{\prime}$ is a subspace of $\mathcal{D}^{\prime}$, the space of distributions. Moreover, each integrable distribution is uniquely determined by its restriction to $C_{0}^{\infty}$. The space $\mathcal{E}^{\prime}$ of compactly supported distributions is a subspace of $\mathcal{D}_{L^{1}}^{\prime}$. Moreover, according to [12], p. 201, each $T \in \mathcal{D}_{L^{1}}^{\prime}$ can be represented as

$$
T=\sum_{\text {finite }} \partial^{\alpha} f_{\alpha}
$$


where $f_{\alpha} \in L^{1}$. As a consequence, we have the (strict) inclusions $\mathcal{E}^{\prime} \subset$ $\mathcal{D}_{L^{1}}^{\prime} \subset \mathcal{S}^{\prime}$. The space $\mathcal{D}_{L^{1}}^{\prime}$ is closed under multiplication by functions in $B$, a fact that we will use repeatedly.

It is possible to consider $\mathcal{D}_{L^{1}}^{\prime}$ as the strong dual of the space $B$, provided that we endow $B$ with a topology that gives rise to the following notion of sequence convergence: A sequence $\left\{\varphi_{j}\right\}$ converges to $\varphi$ if, for each multiindex $\alpha, \sup _{j}\left\|\partial^{\alpha} \varphi_{j}\right\|_{\infty}<\infty$ and the sequence $\left\{\partial^{\alpha} \varphi_{j}\right\}$ converges to $\partial^{\alpha} \varphi$ uniformly on compact sets. If we denote as $B_{c}$ the resulting topological space, it can be proved that $C_{0}^{\infty}$, and so $\dot{B}$, is dense in $B_{c}$ ([12], p. 203). Since every distribution $T \in \mathcal{D}_{L^{1}}^{\prime}$ is well defined on $C_{0}^{\infty}$ and it is continuous on $C_{0}^{\infty}$ with respect to the topology of $B_{c}$, it turns out that $\mathcal{D}_{L^{1}}^{\prime}$ is also the dual of $B_{c}$ ([12], p. 203).

We now use these spaces to define the notion of $\mathcal{S}^{\prime}$-convolution.

Y. Hirata and H. Ogata [7] introduced the $\mathcal{S}^{\prime}$-convolution to extend the validity of the Fourier exchange formula $\mathcal{F}(T * S)=\mathcal{F}(T) \cdot \mathcal{F}(S)$, originally proved by L. Schwartz for pairs of distributions in the Cartesian product $\mathcal{O}_{c}^{\prime} \times \mathcal{S}^{\prime}([12])$. Later, R. Shiraishi proved in [13] an equivalent definition of $\mathcal{S}^{\prime}$-convolution, which is the one we shall use.

Definition 1 ([13]). Given two tempered distributions $T$ and $S$, it is said that the $\mathcal{S}^{\prime}$-convolution of $T$ and $S$ exists if $T(\check{S} * \varphi) \in \mathcal{D}_{L^{1}}^{\prime}$ for every $\varphi \in \mathcal{S}$. When the $\mathcal{S}^{\prime}$-convolution exists, the map

$$
\begin{aligned}
\mathcal{S} & \rightarrow \mathbb{C} \\
\varphi & \rightarrow(T(\check{S} * \varphi), 1)_{\mathcal{D}_{L^{1}}^{\prime}, B_{c}}
\end{aligned}
$$

is linear and continuous. Thus, it defines a tempered distribution which will be denoted by $T * S$.

Here, $T(\check{S} * \varphi)$ is the multiplicative product of the distribution $T$ with the convolution $\breve{S} * \varphi$. This convolution is a smooth function and furthermore, the function and each derivative has at most polynomial growth at infinity.

R. Shiraishi proved in [13] that $T * S$ exists if and only if $S * T$ exists, and they coincide. It is important to remark the fact that Definition 1 coincides with the classical definition in all the cases in which the latter makes sense.

The authors of [1] identified optimal spaces of tempered distributions admitting $\mathcal{S}^{\prime}$-convolution with two possible $n$-dimensional versions of the Poisson kernel: the Euclidean version

$$
P_{y}(x)=\frac{c(n)}{y^{n}} \frac{1}{\left(|x|^{2} / y^{2}+1\right)^{\frac{n+1}{2}}},
$$


where $c(n)=\frac{\Gamma\left(\frac{n+1}{2}\right)}{\pi^{\frac{n+1}{2}}}, y>0$, and the following product domain version

$$
\mathcal{P}_{(y)}(x)=\prod_{i=1}^{n} P_{y_{i}}\left(x_{i}\right)
$$

where $(y)>(0)$, meaning that $y_{1}>0, \ldots, y_{n}>0$. The product domain considered here is the Cartesian product $\mathbb{R}_{+}^{2} \times \ldots \times \mathbb{R}_{+}^{2}$ of $n$ copies of the upper half-plane.

We summarize now these definitions and results.

Definition 2 ([8], [9], [10]). For $x \in \mathbb{R}^{n}$, let $w(x)=\left(1+|x|^{2}\right)^{\frac{1}{2}}$ and let us fix $\mu \in \mathbb{R}$. Then

$$
w^{\mu} \mathcal{D}_{L^{1}}^{\prime}=\left\{T \in \mathcal{S}^{\prime}: w^{-\mu} T \in \mathcal{D}_{L^{1}}^{\prime}\right\}
$$

with the topology induced by the map

$$
\begin{aligned}
w^{\mu} \mathcal{D}_{L^{1}}^{\prime} & \rightarrow \mathcal{D}_{L^{1}}^{\prime} \\
T & \rightarrow w^{-\mu} T .
\end{aligned}
$$

The next theorem characterizes those tempered distributions which are $\mathcal{S}^{\prime}$-convolvable with the classical Poisson kernel $P_{y}$ defined in (2).

Theorem 3 ([1]). Given $T \in \mathcal{S}^{\prime}$, the following statements are equivalent:

(a) $T \in w^{n+1} \mathcal{D}_{L^{1}}^{\prime}$.

(b) $T$ is $\mathcal{S}^{\prime}$-convolvable with $P_{y}$ for each $y>0$.

It is not difficult to see that there is a continuous and strict inclusion $w^{\mu_{1}} \mathcal{D}_{L^{1}}^{\prime} \subset w^{\mu_{2}} \mathcal{D}_{L^{1}}^{\prime}$ when $\mu_{1}<\mu_{2}$. We remark that given $T \in w^{n+1} \mathcal{D}_{L^{1}}^{\prime}$, the $\mathcal{S}^{\prime}$-convolution $T * P_{y}$ is a function defined on $\mathbb{R}^{n}$ whose value at $x$ is given by

$$
\left(w^{-(n+1)}(t) T_{t}, w^{n+1}(t) P_{y}(x-t)\right)_{\mathcal{D}_{L^{1}}^{\prime}, B_{c}}
$$

for each $y>0$ (see [1]). Also, it can be proved ([2]) that the space $w^{n+1} \mathcal{D}_{L^{1}}^{\prime}$ is closed under differentiation, and that if $T \in w^{n+1} \mathcal{D}_{L^{1}}^{\prime}$, then the function $T * P_{y}(x)$ is smooth and $\partial^{\alpha}\left(T * P_{y}\right)=\partial^{\alpha} T * P_{y}$. In fact, we have the following formula to compute the function $T * P_{y}(x)$ :

$$
\left(T * P_{y}\right)(x)=\sum_{\text {finite }}(-1)^{|\alpha|} \int_{\mathbb{R}^{n}} f_{\alpha}(t) \partial_{t}^{\alpha}\left(w^{n+1}(t) P_{y}(x-t)\right) d t,
$$


where $T=\sum_{\text {finite }} w^{n+1} \partial^{\alpha} f_{\alpha}$ with $f_{\alpha} \in L^{1}$ (see [2]). The intrinsic definition given in Definition 1 shows that the formula (4) is independent of the representation of the distribution $T$ as in (1).

The distributions $T$ in the space $w^{\mu} \mathcal{D}_{L^{1}}^{\prime}$ are exactly those tempered distributions that can be represented as

$$
T=\sum_{\text {finite }} w^{\mu} \partial^{\alpha} f_{\alpha}
$$

where $f_{\alpha} \in L^{1}$. In particular, the weighted space $L^{1}\left(w^{-\mu}\right)$ is contained in $w^{\mu} \mathcal{D}_{L^{1}}^{\prime}$. Moreover, the distributions in $w^{\mu} \mathcal{D}_{L^{1}}^{\prime}$ can be written as finite sums of distributional derivatives of functions in $L^{1}\left(w^{-\mu}\right)$ (see [2]).

At this point, it is pertinent to mention that T.M. Flett obtained in $[6$, Th. 6] a necessary and sufficient condition for a measurable function $f$ on $\mathbb{R}^{n}$ to have a well defined Poisson integral, finite everywhere on $\mathbb{R}_{+}^{n+1}$. This condition is exactly that $f \in L^{1}\left(w^{-(n+1)}\right)$. The following lemma extends this observation to the product domain case.

Lemma 4. Given a measurable function $f: \mathbb{R}^{n} \rightarrow \mathbb{C}$, the following statements are equivalent.

(1) The Poisson integral of $f$ is well defined and finite everywhere in $\mathbb{R}_{+}^{2} \times \ldots \times \mathbb{R}_{+}^{2}$.

(2) The Poisson integral of $f$ is well defined and finite at a single point in $\mathbb{R}_{+}^{2} \times \ldots \times \mathbb{R}_{+}^{2}$.

(3) The function $f$ satisfies the integrability condition

$$
\int_{\mathbb{R}^{n}}\left(1+x_{1}^{2}\right)^{\frac{1}{2}} \ldots\left(1+x_{n}^{2}\right)^{\frac{1}{2}}|f(x)| d x<\infty
$$

where $x=\left(x_{1}, \ldots, x_{n}\right)$.

Proof. The proof of this lemma closely resembles the proof of the Euclidean version found in page 762 of [6] and it will be omitted.

Motivated by these arguments, we recall the following definition, which introduces the space of weighted integrable distributions relevant to the product domain case.

Definition $5([1])$. Let $w_{j}=\left(1+x_{j}^{2}\right)^{\frac{1}{2}}, j=1, \ldots, n$. Then

$$
w_{1}^{2} \ldots w_{n}^{2} \mathcal{D}_{L^{1}}^{\prime}=\left\{T \in \mathcal{S}^{\prime}: w_{1}^{-2} \ldots w_{n}^{-2} T \in \mathcal{D}_{L^{1}}^{\prime}\right\}
$$

with the topology induced by the map

$$
w_{1}^{2} \ldots w_{n}^{2} \mathcal{D}_{L^{1}}^{\prime} \rightarrow \mathcal{D}_{L^{1}}^{\prime}
$$




$$
T \rightarrow w_{1}^{-2} \ldots w_{n}^{-2} T
$$

Proposition 13 in [1] shows that neither $w_{1}^{2} \ldots w_{n}^{2} \mathcal{D}_{L^{1}}^{\prime}$ is included in $w^{n+1} \mathcal{D}_{L^{1}}^{\prime}$ nor the other way around. It also shows that both spaces are strictly and continuously included in the space $w^{2 n} \mathcal{D}_{L^{1}}^{\prime}$.

The next result characterizes those tempered distributions that are $\mathcal{S}^{\prime}$ convolvable with the kernel $\mathcal{P}_{(y)}$.

Theorem 6 ([1]). Given $T \in \mathcal{S}^{\prime}$, the following statements are equivalent:

(i) $T \in w_{1}^{2} \ldots w_{n}^{2} \mathcal{D}_{L^{1}}^{\prime}$.

(ii) $T$ is $\mathcal{S}^{\prime}$-convolvable with $\mathcal{P}_{(y)}$ for each $(y)>(0)$.

The proofs of Theorems 3 and 6 use simple representations of distributions in the appropriate spaces as well as estimates involving well chosen test functions in $C_{0}^{\infty}$. The choices of functions in $C_{0}^{\infty}$ imply that the spaces of distributions obtained are optimal for the $\mathcal{S}^{\prime}$-convolution as well as for the notion of convolution that results from requesting that Definition 1 holds only for functions $\varphi$ in $C_{0}^{\infty}$.

Lastly, we introduce the space $\mathcal{D}_{L^{1}}$ of test functions ([12], p. 199).

$$
\mathcal{D}_{L^{1}}=\left\{\varphi \in C^{\infty}: \partial^{\alpha} \varphi \in L^{1} \text { for every multi-index } \alpha\right\},
$$

endowed with the topology defined by the family of norms

$$
\|\varphi\|_{m, 1}=\sum_{|\alpha| \leq m}\left\|\partial^{\alpha} \varphi\right\|_{1}, m=0,1,2, \ldots
$$

The space $\mathcal{D}_{L^{1}}$ is a Fréchet space and we have the dense and continuous strict inclusions $C_{0}^{\infty} \subset \mathcal{D}_{L^{1}} \subset \mathcal{D}^{\prime}$.

We conclude this section stating the following known technical lemma, whose detailed proof can be seen in [2].

Lemma 7. The integral $I(\eta)=\int_{\mathbb{R}^{n}}(1+|\xi|)^{r}(1+|\eta-\xi|)^{s} d \xi$ is finite, for each $\eta \in \mathbb{R}^{n}$, if $r+s+n<0$. Moreover, if this is the case, we have

$$
I(\eta) \leq\left\{\begin{array}{cc}
C_{n, r, s}(1+|\eta|)^{r+s+n} & \text { if } r+n>0 \text { and } s+n>0 \\
C_{n, r, s}(1+|\eta|)^{\max \{r, s\}} & \text { if } \quad r+n<0 \text { or } s+n<0 .
\end{array} .\right.
$$

\section{3. $N$-harmonic extensions of distributions in $w_{1}^{2} \ldots w_{n}^{2} \mathcal{D}_{L^{1}}^{\prime}$}

In this section we obtain $n$-harmonic extensions to the Cartesian product of $n$ copies of the upper half-plane, of distributions in the weighted space $w_{1}^{2} \ldots w_{n}^{2} \mathcal{D}_{L^{1}}^{\prime}$. 
It is shown in [1] that if $T \in \mathcal{D}_{L^{1}}^{\prime}$, the $\mathcal{S}^{\prime}$-convolution with $\mathcal{P}_{(y)}$ is given by the integrable function whose value at $x$ is

$$
\left(T_{t}, \mathcal{P}_{(y)}(x-t)\right)_{\mathcal{D}_{L^{1}}^{\prime}, \dot{B}}
$$

Also, it is proved that this $\mathcal{S}^{\prime}$-convolution defines a function $G=$ $G\left(x_{1}, y_{1} ; \ldots ; x_{n}, y_{n}\right)$ that is smooth and $n$-harmonic in the product domain $\mathbb{R}_{+}^{2} \times \ldots \times \mathbb{R}_{+}^{2}$. In other words, $G$ is a harmonic function of each pair $\left(x_{j}, y_{j}\right)$ separately $([4])$. Moreover, ([4], [1]) $T * \mathcal{P}_{(y)}$ converges to $T$ in $\mathcal{D}_{L^{1}}^{\prime}$ as $(y) \rightarrow(0)^{+}$, which means $y_{j} \rightarrow 0^{+}$for each $j=1, \ldots, n$. These results imply that we can construct $n$-harmonic extensions of distributions in $\mathcal{D}_{L^{1}}^{\prime}$ by considering the $\mathcal{S}^{\prime}$-convolution with the kernel $\mathcal{P}_{(y)}$.

In this section we will obtain these results for distributions in the weighted space $w_{1}^{2} \ldots w_{n}^{2} \mathcal{D}_{L^{1}}^{\prime}$

First, we need to find a convenient formula to compute the $\mathcal{S}^{\prime}$-convolution of a distribution $T \in w_{1}^{2} \ldots w_{n}^{2} \mathcal{D}_{L^{1}}^{\prime}$ with the kernel $\mathcal{P}_{(y)}$. We claim that this convolution coincides with the function whose value at $x$ is given by

$$
\left(w_{1}^{-2}\left(t_{1}\right) \ldots w_{n}^{-2}\left(t_{n}\right) T_{t}, \prod_{j=1}^{n} w_{j}^{2}\left(t_{j}\right) P_{y_{j}}\left(x_{j}-t_{j}\right)\right)_{\mathcal{D}_{L^{1}}^{\prime}, B_{c}}
$$

for each $(y)>(0)$. Indeed, if we write

$$
\prod_{j=1}^{n} w_{j}^{-2} T=\sum_{\text {finite }} \partial^{\alpha} f_{\alpha}
$$

with $f_{\alpha} \in L^{1}\left(\mathbb{R}^{n}\right)$, we know that for $\varphi \in \mathcal{S}$

$$
\left(T * \mathcal{P}_{(y)}, \varphi\right)_{\mathcal{S}^{\prime}, \mathcal{S}}=\left(w_{1}^{-2} \ldots w_{n}^{-2} T w_{1}^{2} \ldots w_{n}^{2}\left(\stackrel{\vee}{\mathcal{P}}_{(y)} * \varphi\right), 1\right)_{\mathcal{D}_{L^{1}}^{\prime}, B_{c}} .
$$

We next notice that the function $w_{1}^{2} \ldots w_{n}^{2}\left(\stackrel{\vee}{\mathcal{P}}_{(y)} * \varphi\right)$ belongs to $B$. In fact, there exists a constant $C>0$ not depending on $(y)$ such that

$$
\begin{aligned}
\left|w_{1}^{2} \ldots w_{n}^{2}\left(\stackrel{\vee}{\mathcal{P}}_{(y)} * \varphi\right)(x)\right| \leq & \frac{C}{y_{1} \ldots y_{n}} \prod_{j=1}^{n}\left(1+x_{j}^{2}\right)\left(1+\frac{x_{j}^{2}}{y_{j}^{2}}\right)^{-1} \\
& \times\left[\|\varphi\|_{L^{1}\left(\mathbb{R}^{n}\right)}+\sum \frac{1}{y_{i_{1}}^{2} \ldots y_{i_{k}}^{2}}\left\|t_{i_{1}}^{2} \ldots t_{i_{k}}^{2} \varphi\right\|_{L^{1}\left(\mathbb{R}^{n}\right)}\right],
\end{aligned}
$$

where the sum is taken over all the possible $k$-tuples $\left(i_{1}, \ldots, i_{k}\right)$ with $1 \leq i_{1}<\ldots<i_{k} \leq n, 1 \leq k \leq n$. Thus, the right hand side of (8) can be expanded using Fubini's theorem to obtain the claimed result. 
One can prove, as in the Euclidean case (see [2]), the following characterization for the space $w_{1}^{2} \ldots w_{n}^{2} \mathcal{D}_{L^{1}}^{\prime}$.

\section{Lemma 8.}

$$
w_{1}^{2} \ldots w_{n}^{2} \mathcal{D}_{L^{1}}^{\prime}=\left\{T \in \mathcal{S}^{\prime}: T=\sum_{\text {finite }} \partial^{\alpha} f_{\alpha}, f_{\alpha} \in L^{1}\left(w_{1}^{-2} \ldots w_{n}^{-2}\right)\right\} .
$$

We can also obtain the following result.

Lemma 9. The space $w_{1}^{2} \ldots w_{n}^{2} \mathcal{D}_{L^{1}}^{\prime}$ is closed under differentiation. Moreover, if $T \in w_{1}^{2} \ldots w_{n}^{2} \mathcal{D}_{L^{1}}^{\prime}$ then $\partial^{\alpha}\left(T * \mathcal{P}_{(y)}\right)=\left(\partial^{\alpha} T\right) * \mathcal{P}_{(y)}$ for every multi-index $\alpha$.

Proof. The first assertion is immediate using Lemma 8. To prove the second assertion, it suffices to consider test functions $\varphi$ of the form $\varphi_{1}\left(x_{1}\right) \ldots \varphi_{n}\left(x_{n}\right)$, with $\varphi_{i} \in \mathcal{S}(\mathbb{R})$, since the space $\underbrace{\mathcal{S}(\mathbb{R}) \otimes \ldots \otimes \mathcal{S}(\mathbb{R})}_{\mathrm{n} \text { times }}$ is dense in $\mathcal{S}$.

Let us fix a function $\psi \in C_{0}^{\infty}(\mathbb{R})$ such that $0 \leq \psi \leq 1, \psi=1$ in $|x| \leq 1$ and $\psi=0$ in $|x| \geq 2$, and let us set $\psi_{j}(x)=\psi\left(x_{1} / j\right) \ldots \psi\left(x_{n} / j\right)$ for each $j=1,2, \ldots$. We first observe that

$$
\mathcal{P}_{(y)} * \varphi=\prod_{i=1}^{n} P_{y_{i}} * \varphi_{i} .
$$

Next we write $T=w_{1}^{2} \ldots w_{n}^{2} S$, with $S \in \mathcal{D}_{L^{1}}^{\prime}$. Thus

$$
\begin{aligned}
& \left(\left(\mathcal{P}_{(y)} * \varphi\right) \partial^{\alpha} T, \psi_{j}\right)_{\mathcal{D}_{L^{1}}^{\prime}, B_{c}} \\
& =(-1)^{|\alpha|}\left(T, \prod_{i=1}^{n} \psi\left(x_{i} / j\right)\left(P_{y_{i}} * \partial^{\alpha_{i}} \varphi_{i}\right)\right)_{\mathcal{S}^{\prime}, \mathcal{S}} \\
& +(-1)^{|\alpha|} C_{\gamma, \alpha} \sum_{0<\gamma \leq \alpha}\left(S, \prod_{i=1}^{n} w_{i}^{2} \partial^{\gamma_{i}} \psi\left(x_{i} / j\right)\left(P_{y_{i}} * \partial^{\alpha_{i}-\gamma_{i}} \varphi_{i}\right)\right)_{\mathcal{D}_{L^{1}}^{\prime}, B_{c}} .
\end{aligned}
$$

For each $i=1,2, \ldots, \psi\left(x_{i} / j\right) \rightarrow 1$ in $B_{c}(\mathbb{R})$ as $j \rightarrow \infty$. Moreover, for each $y_{i}>0$ there exists $C=C_{y_{i}}>0$ such that $\left|P_{y_{i}} * \partial^{\alpha_{i}-\gamma_{i}} \varphi_{i}\right| \leq C_{y_{i}} w_{i}^{-2}$. Then, $\prod_{i=1}^{n} w_{i}^{2} \partial^{\gamma_{i}} \psi\left(x_{i} / j\right)\left(P_{y_{i}} * \partial^{\alpha_{i}-\gamma_{i}} \varphi_{i}\right) \rightarrow 0$ in $B_{c}$ as $j \rightarrow \infty$. Thus (9) $\left(\left(\partial^{\alpha} T\right) * \mathcal{P}_{(y)}, \varphi\right)_{\mathcal{S}^{\prime}, \mathcal{S}}=\lim _{j \rightarrow \infty}(-1)^{|\alpha|}\left(T, \prod_{i=1}^{n} \psi\left(x_{i} / j\right)\left(P_{y_{i}} * \partial^{\alpha_{i}} \varphi_{i}\right)\right)_{\mathcal{S}^{\prime}, \mathcal{S}}$. 
Finally, we can see that the right-hand side of (9) coincides with $\left(\partial^{\alpha}\left(T * \mathcal{P}_{(y)}\right), \varphi\right)_{\mathcal{S}^{\prime}, \mathcal{S}}$, therefore

$$
\left(\left(\partial^{\alpha} T\right) * \mathcal{P}_{(y)}, \varphi\right)_{\mathcal{S}^{\prime}, \mathcal{S}}=\left(\partial^{\alpha}\left(T * \mathcal{P}_{(y)}\right), \varphi\right)_{\mathcal{S}^{\prime}, \mathcal{S}}
$$

for each $\varphi \in \mathcal{S}(\mathbb{R}) \otimes \ldots \otimes \mathcal{S}(\mathbb{R})$. Then $\partial^{\alpha}\left(T * \mathcal{P}_{(y)}\right)=\left(\partial^{\alpha} T\right) * \mathcal{P}_{(y)}$. This completes the proof of Lemma 9.

Lemma 10. Given $T \in w_{1}^{2} \ldots w_{n}^{2} \mathcal{D}_{L^{1}}^{\prime}$ the $\mathcal{S}^{\prime}$-convolution $T * \mathcal{P}_{(y)}$ belongs to the space $w_{1}^{2} \ldots w_{n}^{2} \mathcal{D}_{L^{1}}$ for each $(y)>(0)$.

Proof. According to Lemma 9, it is enough to prove that $S * \mathcal{P}_{(y)} \in$ $L^{1}\left(w_{1}^{-2} \ldots w_{n}^{-2}\right)$ for every $S \in w_{1}^{2} \ldots w_{n}^{2} \mathcal{D}_{L^{1}}^{\prime}$.

Without loss of generality we assume that $w_{1}^{-2} \ldots w_{n}^{-2} S=\partial^{\alpha} f$, with $f \in L^{1}$. Then, according to (7) we have

$$
\begin{aligned}
S * \mathcal{P}_{(y)}(x) & =\left(w_{1}^{-2}\left(t_{1}\right) \ldots w_{n}^{-2}\left(t_{n}\right) S_{t}, \prod_{j=1}^{n} w_{j}^{2}\left(t_{j}\right) P_{y_{j}}\left(x_{j}-t_{j}\right)\right)_{\mathcal{D}_{L^{1}}^{\prime}, B_{c}} \\
& =\sum_{\beta \leq \alpha}(-1)^{|\alpha|} C_{\alpha, \beta} \int_{\mathbb{R}^{n}} f(t) \prod_{j=1}^{n} \partial_{t_{j}}^{\beta_{j}} w_{j}^{2}\left(t_{j}\right) \partial_{t_{j}}^{\alpha_{j}-\beta_{j}} P_{y_{j}}\left(x_{j}-t_{j}\right) d t .
\end{aligned}
$$

For each $j=1, \ldots, n$ we have

$$
\left|\partial^{\beta_{j}} w_{j}^{2}\left(t_{j}\right)\right| \leq C_{\beta_{j}}\left(1+t_{j}^{2}\right)^{\frac{2-\beta_{j}}{2}}
$$

and

$$
\left|\partial^{\alpha_{j}-\beta_{j}} P_{y_{j}}\left(x_{j}-t_{j}\right)\right| \leq \frac{C_{\alpha_{j}, \beta_{j}}}{y_{j}^{1+\alpha_{j}-\beta_{j}}}\left(1+\frac{\left(x_{j}-t_{j}\right)^{2}}{y_{j}^{2}}\right)^{-\frac{\left(2+\left(\alpha_{j}-\beta_{j}\right)\right)}{2}} .
$$

Then we have that

$$
\begin{aligned}
\left|S * \mathcal{P}_{(y)}(x)\right| \leq & \sum_{\beta \leq \alpha} \frac{C_{\alpha, \beta}}{\prod_{1 \leq j \leq n} y_{j}^{1+\alpha_{j}-\beta_{j}}} \int_{\mathbb{R}^{n}}|f(t)| \prod_{j=1}^{n}\left(1+t_{j}^{2}\right)^{\frac{2-\beta_{j}}{2}} \\
& \times\left(1+\frac{\left(x_{j}-t_{j}\right)^{2}}{y_{j}^{2}}\right)^{-\frac{\left(2+\left(\alpha_{j}-\beta_{j}\right)\right)}{2}} d t .
\end{aligned}
$$

Thus

$$
\int_{\mathbb{R}^{n}}\left|S * \mathcal{P}_{(y)}(x)\right| w_{1}^{-2}\left(x_{1}\right) \ldots w_{n}^{-2}\left(x_{n}\right) d x \leq \sum_{\beta \leq \alpha} \frac{C_{\alpha, \beta}}{\prod_{1 \leq j \leq n} y_{j}^{1+\alpha_{j}-\beta_{j}}} J_{\alpha \beta},
$$


where

$$
\begin{aligned}
J_{\alpha, \beta}= & \int_{\mathbb{R}^{n}}|f(t)|\left[\prod_{j=1}^{n} \int_{-\infty}^{\infty} w_{j}^{-2}\left(x_{j}\right)\left(1+\frac{\left(x_{j}-t_{j}\right)^{2}}{y_{j}^{2}}\right)^{-\frac{\left(2+\left(\alpha_{j}-\beta_{j}\right)\right)}{2}} d x_{j}\right] \\
& \times \prod_{j=1}^{n}\left(1+t_{j}^{2}\right)^{\frac{2-\beta_{j}}{2}} d t .
\end{aligned}
$$

Now, applying Lemma 7

$$
\begin{aligned}
& \prod_{j=1}^{n} \int_{-\infty}^{\infty} w_{j}^{-2}\left(x_{j}\right)\left(1+\frac{\left(x_{j}-t_{j}\right)^{2}}{y_{j}^{2}}\right)^{-\frac{\left(2+\left(\alpha_{j}-\beta_{j}\right)\right)}{2}} d x_{j} \\
& \leq C_{\alpha, \beta,(y)} \prod_{j=1}^{n}\left(1+\left|t_{j}\right|\right)^{-2} .
\end{aligned}
$$

So

$$
J_{\alpha, \beta} \leq C_{\alpha, \beta,(y)} \int_{\mathbb{R}^{n}}|f(t)| \prod_{j=1}^{n}\left(1+\left|t_{j}\right|\right)^{-2}\left(1+\left|t_{j}\right|\right)^{2-\beta_{j}} d t<\infty .
$$

This concludes the proof of Lemma 10.

We are now ready to prove the main result in this section, namely

Theorem 11. Given $T \in w_{1}^{2} \ldots w_{n}^{2} \mathcal{D}_{L^{1}}^{\prime}$, the $\mathcal{S}^{\prime}$-convolution $T * \mathcal{P}_{(y)}$ converges to $T$ in $w_{1}^{2} \ldots w_{n}^{2} \mathcal{D}_{L^{1}}^{\prime}$ as $(y) \rightarrow(0)^{+}$.

Proof. According to Lemma $10, T * \mathcal{P}_{(y)} \in w_{1}^{2} \ldots w_{n}^{2} \mathcal{D}_{L^{1}} \subset w_{1}^{2} \ldots w_{n}^{2} \mathcal{D}_{L^{1}}^{\prime}$ for each $(y)>(0)$. We must show that for every bounded subset $A$ of $\dot{B}$,

$$
\left(w_{1}^{-2} \ldots w_{n}^{-2}\left(T * \mathcal{P}_{(y)}\right), \varphi\right)_{\mathcal{D}_{L^{1}}^{\prime}, \dot{B}} \rightarrow\left(w_{1}^{-2} \ldots w_{n}^{-2} T, \varphi\right)_{\mathcal{D}_{L^{1}}^{\prime}, \dot{B}}
$$

uniformly with respect to $\varphi \in A$.

Without loss of generality let us assume that $w_{1}^{-2} \ldots w_{n}^{-2} T=\partial^{\alpha} f$ for some $f \in L^{1}$. If we fix $\varphi \in A$ we can write

$$
\begin{aligned}
& \left(w_{1}^{-2} \ldots w_{n}^{-2}\left(T * \mathcal{P}_{(y)}\right), \varphi\right)_{\mathcal{D}_{L^{1}}^{\prime}, \dot{B}} \\
& =(-1)^{|\alpha|} \int_{\mathbb{R}^{n}} \int_{\mathbb{R}^{n}} f(t)\left[\sum_{\beta \leq \alpha} C_{\alpha, \beta} \prod_{j=1}^{n} \partial^{\beta_{j}} w_{j}^{2}\left(t_{j}\right) \partial_{x_{j}}^{\alpha_{j}-\beta_{j}} P_{y_{j}}\left(x_{j}-t_{j}\right) d t\right] \\
& \quad \times\left(\prod_{j=1}^{n} w_{j}^{-2}\left(x_{j}\right)\right) \varphi(x) d x
\end{aligned}
$$




$$
\begin{aligned}
= & \int_{\mathbb{R}^{n}} \int_{\mathbb{R}^{n}} f(t)\left[\sum_{\beta \leq \alpha} C_{\alpha, \beta} \prod_{j=1}^{n} \partial^{\beta_{j}} w_{j}^{2}\left(t_{j}\right)(-1)^{|\beta|} \partial_{x_{j}}^{\alpha_{j}-\beta_{j}} P_{y_{j}}\left(x_{j}-t_{j}\right) d t\right] \\
& \times\left(\prod_{j=1}^{n} w_{j}^{-2}\left(x_{j}\right)\right) \varphi(x) d x .
\end{aligned}
$$

We claim that we can change the order of integration to obtain

$$
\begin{aligned}
& \sum_{\beta \leq \alpha} C_{\alpha, \beta} \int_{\mathbb{R}^{n}} f(t)\left[(-1)^{|\beta|} \int_{\mathbb{R}^{n}} \partial_{x}^{\alpha-\beta}\left(\mathcal{P}_{(y)}(x-t)\right)\left(\prod_{j=1}^{n} w_{j}^{-2}\left(x_{j}\right)\right) \varphi(x) d x\right] \\
& \quad \times \partial^{\beta}\left(\prod_{j=1}^{n} w_{j}^{2}\left(t_{j}\right)\right) d t= \\
& =\sum_{\beta \leq \alpha}(-1)^{|\alpha|} C_{\alpha, \beta} \int_{\mathbb{R}^{n}} f(t)\left[\int_{\mathbb{R}^{n}} \mathcal{P}_{(y)}(x-t) \partial^{\alpha-\beta}\left(\left(\prod_{j=1}^{n} w_{j}^{-2}\left(x_{j}\right)\right) \varphi(x)\right) d x\right] \\
& \quad \times \partial^{\beta}\left(\prod_{j=1}^{n} w_{j}^{2}\left(t_{j}\right)\right) d t
\end{aligned}
$$

Indeed, if we denote

$$
s_{|\alpha|}(\varphi)=\sup _{0 \leq|\gamma| \leq|\alpha|}\left\|\partial^{\gamma} \varphi\right\|_{\infty},
$$

then, according to Lemma 7 we can estimate

$$
\begin{aligned}
& \int_{\mathbb{R}^{n}} \mathcal{P}_{(y)}(x-t)\left|\partial^{\alpha-\beta}\left(\left(\prod_{j=1}^{n} w_{j}^{-2}\left(x_{j}\right)\right) \varphi(x)\right)\right| d x \\
& \leq C_{\alpha, \beta,(y)} s_{|\alpha|}(\varphi) \prod_{j=1}^{n} \int_{-\infty}^{\infty} P_{y_{j}}\left(x_{j}-t_{j}\right) w_{j}^{-2}\left(x_{j}\right) d x_{j} \\
& \leq C_{\alpha, \beta,(y)} s_{|\alpha|}(\varphi) \prod_{j=1}^{n} w_{j}^{-2}\left(t_{j}\right)
\end{aligned}
$$

and this implies that

$$
\begin{aligned}
& \int_{\mathbb{R}^{n}} \int_{\mathbb{R}^{n}}|f(t)| \mathcal{P}_{(y)}(x-t)\left|\partial^{\alpha-\beta}\left(\left(\prod_{j=1}^{n} w_{j}^{-2}\left(x_{j}\right)\right) \varphi(x)\right)\right| \\
& \times\left|\partial^{\beta}\left(\prod_{j=1}^{n} w_{j}^{2}\left(t_{j}\right)\right)\right| d x d t
\end{aligned}
$$


is finite.

Now, to prove (10) it suffices to show that

$$
\int_{\mathbb{R}^{n}}|f(t)|\left|\Phi_{(y)}(t)-\partial^{\alpha} \varphi(t)\right| d t \rightarrow 0
$$

as $(y) \rightarrow(0)^{+}$, uniformly with respect to $\varphi \in A$, where

$$
\begin{aligned}
\Phi_{(y)}(t)= & \sum_{\beta \leq \alpha} C_{\alpha, \beta}\left[\int_{\mathbb{R}^{n}} \mathcal{P}_{(y)}(x-t) \partial^{\alpha-\beta}\left(\left(\prod_{j=1}^{n} w_{j}^{-2}\left(x_{j}\right)\right) \varphi(x)\right) d x\right] \\
& \times \partial^{\beta}\left(\prod_{j=1}^{n} w_{j}^{2}\left(t_{j}\right)\right) .
\end{aligned}
$$

So, let us prove (11). For $M>0$ to be chosen later, we can write

$$
\begin{aligned}
& \int_{\mathbb{R}^{n}}|f(t)|\left|\Phi_{(y)}(t)-\partial^{\alpha} \varphi(t)\right| d t \\
& =\int_{|t| \leq M}|f(t)|\left|\Phi_{(y)}(t)-\partial^{\alpha} \varphi(t)\right| d t+\int_{|t| \geq M}|f(t)|\left|\Phi_{(y)}(t)-\partial^{\alpha} \varphi(t)\right| d t \\
& =I_{1}+I_{2} .
\end{aligned}
$$

Given $\varepsilon>0$, we claim that we can choose $M$ so that

$$
I_{2}<C_{A} \varepsilon
$$

for every $\varphi \in A$ and each $(0)<(y)<(1)$, meaning $0<y_{j}<1$ for every $j$. To prove this claim we can write

$$
\begin{aligned}
& \left|\Phi_{(y)}(t)-\partial^{\alpha} \varphi(t)\right| \\
& \leq \sum_{\beta \leq \alpha} C_{\alpha, \beta} \int_{\mathbb{R}^{n}}\left[\mathcal{P}_{(y)}(x-t) \mid \partial^{\alpha-\beta}\left(\left(\prod_{j=1}^{n} w_{j}^{-2}\right) \varphi(x)\right)\right. \\
& \left.\quad-\partial^{\alpha-\beta}\left(\left(\prod_{j=1}^{n} w_{j}^{-2}\right) \varphi(t)\right) \| \partial^{\beta}\left(\prod_{j=1}^{n} w_{j}^{2}\left(t_{j}\right)\right) \mid\right] d x \\
& \leq C_{\alpha} s_{|\alpha|}(\varphi)\left[\int_{\mathbb{R}^{n}} \mathcal{P}_{(y)}(x-t) \prod_{j=1}^{n} w_{j}^{-2}(x) d x\right. \\
& \left.\quad+\prod_{j=1}^{n} w_{j}^{-2}(t) \int_{\mathbb{R}^{n}} \mathcal{P}_{(y)}(x-t) d x\right] \prod_{j=1}^{n} w_{j}^{2}\left(t_{j}\right)
\end{aligned}
$$




$$
\begin{gathered}
=C_{\alpha} s_{|\alpha|}(\varphi)\left[\prod_{j=1}^{n} \int_{-\infty}^{\infty} P_{y_{j}}\left(x_{j}-t_{j}\right) w_{j}^{-2}\left(x_{j}\right) d x_{j}\right. \\
\left.+\prod_{j=1}^{n} w_{j}^{-2}(t) \int_{-\infty}^{\infty} P_{y_{j}}\left(x_{j}-t_{j}\right) d x_{j}\right] \prod_{j=1}^{n} w_{j}^{2}\left(t_{j}\right) .
\end{gathered}
$$

Now, we observe that

$$
\int_{-\infty}^{\infty} P_{y_{j}}\left(x_{j}-t_{j}\right) w_{j}^{-2}\left(x_{j}\right) d x_{j}=C\left(P_{y_{j}} * P_{1}\right)\left(t_{j}\right)=C P_{1+y_{j}}\left(t_{j}\right),
$$

where $C$ does not depend on $(y)$. Thus

$$
\left|\Phi_{(y)}(t)-\partial^{\alpha} \varphi(t)\right| \leq C_{\alpha} s_{|\alpha|}(\varphi)\left[1+\prod_{j=1}^{n} P_{1+y_{j}}\left(t_{j}\right) w_{j}^{2}\left(t_{j}\right)\right] \leq C_{\alpha} s_{|\alpha|}(\varphi) .
$$

Then

$$
I_{2} \leq C_{\alpha} s_{|\alpha|}(\varphi) \int_{|t| \geq M}|f(t)| d t
$$

Since the set $A$ is bounded in $\dot{B}, \sup _{\varphi \in A} s_{|\alpha|}(\varphi)=K_{A}<\infty$. On the other hand, we can choose $M$ large enough so that

$$
\int_{|t| \geq M}|f(t)| d t<\varepsilon
$$

and this concludes the proof of (12).

Next, we need to estimate $I_{1}$ for the value of $M$ previously selected.

First, we write

$$
\begin{aligned}
& \int_{\mathbb{R}^{n}} \mathcal{P}_{(y)}(x-t)\left|\partial^{\alpha-\beta}\left(\left(\prod_{j=1}^{n} w_{j}^{-2}\right) \varphi(x)\right)-\partial^{\alpha-\beta}\left(\left(\prod_{j=1}^{n} w_{j}^{-2}\right) \varphi(t)\right)\right| d x \\
& =\int_{|x-t|<1}+\int_{|x-t|>1} \\
& =J_{1}+J_{2} .
\end{aligned}
$$

Now, observe that $|x-t|>1$ implies $\left|x_{i}-t_{i}\right|>1 / 2$ for some $i$, that is, $x \in \cup_{i=1}^{n}\left\{x:\left|x_{i}-t_{i}\right|>1 / 2\right\}$. Thus

$$
J_{2} \leq C_{\alpha \beta} s_{|\alpha|}(\varphi) \sum_{i=1}^{n} \int_{\left\{x:\left|x_{i}-t_{i}\right|>1 / 2\right.} \prod_{j=1}^{n} P_{y_{j}}\left(x_{j}-t_{j}\right) d x
$$




$$
\begin{aligned}
\leq & C_{\alpha \beta} s_{|\alpha|}(\varphi) \sum_{i=1}^{n} \int_{-\infty}^{\infty} P_{y_{1}}\left(x_{1}-t_{1}\right) d x_{1} \ldots \int_{\left|x_{i}-t_{i}\right|>1 / 2} P_{y_{i}}\left(x_{i}-t_{i}\right) d x_{i} \ldots \\
& \times \int_{-\infty}^{\infty} P_{y_{n}}\left(x_{n}-t_{n}\right) d x_{n} \\
= & C_{\alpha \beta} s_{|\alpha|}(\varphi) \sum_{i=1}^{n} \int_{\left|x_{i}-t_{i}\right|>1 / 2} P_{y_{i}}\left(x_{i}-t_{i}\right) d x_{i} \\
\leq & C_{\alpha \beta} s_{|\alpha|}(\varphi) \sum_{i=1}^{n} \int_{\left|u_{i}\right|>1 / 2 y_{i}}\left(1+u_{i}^{2}\right)^{-1} d u_{i} .
\end{aligned}
$$

Concerning $J_{1}$ we can write

$$
\begin{aligned}
J_{1}= & \int_{|x-t|<1} \mathcal{P}_{(y)}(x-t) \mid \int_{0}^{1} \nabla\left(\partial^{\alpha-\beta}\left(w_{1}^{-2} \ldots w_{n}^{-2} \varphi\right)\right) \\
& \times(t+\theta(x-t)) \cdot(x-t) d \theta \mid d x \\
\leq & C_{\alpha, \beta} s_{|\alpha|+1}(\varphi) \int_{|x-t|<1} \mathcal{P}_{(y)}(x-t)|x-t| d x .
\end{aligned}
$$

Moreover, for any $0<\sigma<1$ we have

$$
\begin{aligned}
& \int_{|x-t|<1} \mathcal{P}_{(y)}(x-t)|x-t| d x \\
\leq & \sum_{j=1}^{n} \int_{|x-t|<1} \mathcal{P}_{(y)}(x-t)\left|x_{j}-t_{j}\right| d x \\
\leq & \sum_{j=1}^{n} \int_{\left\{x:\left|x_{i}-t_{i}\right|<1\right.} \mathcal{P}_{(y)}(x-t)\left|x_{j}-t_{j}\right| d x \\
\leq & \sum_{j=1}^{n} \int_{\left|x_{1}-t_{1}\right|<1} P_{y_{1}}\left(x_{1}-t_{1}\right) d x_{1} \ldots \int_{\left|x_{j}-t_{j}\right|<1} P_{y_{j}}\left(x_{j}-t_{j}\right)\left|x_{j}-t_{j}\right| d x_{j} \ldots \\
& \times \int_{\left|x_{n}-t_{n}\right|<1} P_{y_{n}}\left(x_{n}-t_{n}\right) d x_{n} \\
\leq & \sum_{j=1}^{n} \int_{\left|x_{j}-t_{j}\right|<1} P_{y_{j}}\left(x_{j}-t_{j}\right)\left|x_{j}-t_{j}\right| d x_{j} \\
\leq & \sum_{j=1}^{n} \frac{C}{y_{j}} \int_{\left|x_{j}-t_{j}\right|<1}\left(1+\frac{\left|x_{j}-t_{j}\right|}{y_{j}}\right)^{-2}\left|x_{j}-t_{j}\right|^{\sigma} d x_{j} \\
\leq & \sum_{j=1}^{n} C y_{j}^{\sigma} \int_{-\infty}^{\infty}\left(1+\left|u_{j}\right|\right)^{-2}\left|u_{j}\right|^{\sigma} d u_{j}=C \sum_{j=1}^{n} y_{j}^{\sigma},
\end{aligned}
$$


where $C$ does not depend on $(y)$.

Since $\sup _{\varphi \in A} s_{|\alpha|+1}(\varphi)=H_{A}<\infty$ we obtain the estimate

$$
I_{1} \leq C\left[H_{A} \sum_{j=1}^{n} y_{j}^{\sigma}+K_{A} \sum_{i=1}^{n} \int_{\left|u_{i}\right|>1 / 2 y_{i}}\left(1+u_{i}^{2}\right)^{-1} d u_{i}\right] \int_{|t| \leq M}|f(t)| d t
$$

Finally, gathering estimates (13), (14) and (15) we can write

$$
\begin{aligned}
& \int_{\mathbb{R}^{n}}\left|f(t) \| \Phi_{(y)}(t)-\partial^{\alpha} \varphi(t)\right| d t \\
& \leq C_{\alpha}\left[K_{A} \varepsilon+\|f\|_{1}\left(H_{A} \sum_{j=1}^{n} y_{j}^{\sigma}+K_{A} \sum_{i=1}^{n} \int_{\left|u_{i}\right|>1 / 2 y_{i}}\left(1+u_{i}^{2}\right)^{-1} d u_{i}\right)\right]
\end{aligned}
$$

for each $(0)<(y)<(1)$. As a consequence,

$$
\limsup _{(y) \rightarrow(0)^{+}} \int_{\mathbb{R}^{n}}|f(t)|\left|\Phi_{(y)}(t)-\partial^{\alpha} \varphi(t)\right| d t \leq C_{\alpha} K_{A} \varepsilon
$$

for every $\varepsilon>0$, proving in this way (11).

This concludes the proof of Theorem 11 .

Remark 12. By Theorem 11, every distribution $T \in w_{1}^{2} \ldots w_{n}^{2} \mathcal{D}_{L^{1}}^{\prime}$ has a $n$-harmonic extension to $\mathbb{R}_{+}^{2} \times \ldots \times \mathbb{R}_{+}^{2}$ given by the $\mathcal{S}^{\prime}$-convolution $T * \mathcal{P}_{(y)}(x)$. This extension is not unique, since the $n$-harmonic function $y_{1} \ldots y_{n}$ extends the zero distribution.

\section{A characterization for a class of $n$-harmonic functions.}

Before stating this characterization, we need to introduce some relevant notation. We set

$$
\begin{aligned}
W & =W(x, y) \stackrel{\text { def }}{=}\left(1+|x|^{2}+y^{2}\right)^{1 / 2}, \\
W_{j} & =W_{j}\left(x_{j}, y_{j}\right) \stackrel{\text { def }}{=}\left(1+x_{j}^{2}+y_{j}^{2}\right)^{1 / 2} \text { for } j=1, \ldots, n
\end{aligned}
$$

where $(x, y) \in \mathbb{R}_{+}^{n+1}$. We also indicate

$$
\mathcal{P}_{\left(y_{i_{1}}, \ldots, y_{i_{k}}\right)}=P_{y_{i_{1}}} \ldots P_{y_{i_{k}}} .
$$

With $L^{1, \infty}\left(\mathbb{R}_{+}^{n+1}\right)$ we denote the linear space of functions $f$ that satisfy the weak-type inequality

$$
\operatorname{meas}\left(\left\{(x, y) \in \mathbb{R}_{+}^{n+1}:|f(x, y)|>\lambda\right\}\right) \leq \frac{C}{\lambda},
$$


for some $C>0$ and for every $\lambda>0$, where meas means the Lebesgue measure in $\mathbb{R}_{+}^{n+1}$. The space $L^{1, \infty}\left(\mathbb{R}_{+}^{n+1}\right)$ is endowed with the quasi-norm

$$
\|f\|_{1, \infty}=\inf \{C>0: C \text { satisfies (16) }\} .
$$

Theorem 13. Let $u: \mathbb{R}_{+}^{2} \times \ldots \times \mathbb{R}_{+}^{2} \rightarrow \mathbb{C}$ be an $n$-harmonic function. Then the following two statements are equivalent:

(1) The function $u=u\left(z_{1}, \ldots, z_{n}\right)$ where $z_{j}=\left(x_{j}, y_{j}\right)$, can be written as a finite sum of functions of the form

$$
\partial_{x_{1}}^{\alpha_{1}} G_{\alpha_{1}}\left(z_{1}\right) \ldots \partial_{x_{n}}^{\alpha_{n}} G_{\alpha_{n}}\left(z_{n}\right)
$$

where the function $G_{\alpha_{i}}$ is harmonic in $\mathbb{R}_{+}^{2}$ and $W_{i}^{-2} y_{i}^{-1} G_{\alpha_{i}}$ belongs to $L^{1, \infty}\left(\mathbb{R}_{+}^{2}\right), i=1, \ldots, n$.

(2) The function $u$ can be represented as

$$
u=\sum_{I \subset\{1,2, \ldots, n\}} c_{I} y_{i_{1}} \ldots y_{i_{k}}\left(T_{j_{1}, \ldots, j_{n-k}} * \mathcal{P}_{\left(y_{j_{1}}, \ldots, y_{j_{n-k}}\right)}\right),
$$

where for each pair of sets $I=\left\{i_{1}, \ldots, i_{k}\right\},\left\{j_{1}, \ldots, j_{n-k}\right\}=$ $\{1,2, \ldots, n\} \backslash I$,

$$
T_{j_{1}, \ldots, j_{n-k}}=T_{j_{1}} \otimes \ldots \otimes T_{j_{n-k}}
$$

with $T_{j_{l}}$ in $w_{j_{l}}^{2} \mathcal{D}_{L^{1}}^{\prime}(\mathbb{R})$, and $c_{I} \in \mathbb{C}$.

This characterization relies on the following theorem by P. Sjögren [14].

Theorem 14 ([14]). Let $u: \mathbb{R}_{+}^{n+1} \rightarrow \mathbb{C}$ be a harmonic function. Then the following two statements are equivalent:

(1) The function $W^{-n-1} y^{-1} u$ belongs to $L^{1, \infty}\left(\mathbb{R}_{+}^{n+1}\right)$.

(2) There exists a complex Borel measure $\mu$ satisfying

$$
\int_{\mathbb{R}^{n}} w^{-n-1}(x) d|\mu|(x)<\infty
$$

and there exists $a \in \mathbb{C}$ such that

$$
u=\mu * P_{y}+a y .
$$

Moreover, the quantities $\int_{\mathbb{R}^{n}} w^{-n-1}(x) d|\mu|(x)+|a|$ and $\left\|W^{-n-1} y^{-1} u\right\|_{1, \infty}$ are equivalent.

We now prove Theorem 13. 
Proof. We first prove that 1) implies 2). According to the onedimensional version of Theorem 14, for each multi-index $\alpha$ there exist a measure $\mu_{\alpha_{j}}$ satisfying (18) and $c_{\alpha_{j}} \in \mathbb{C}$ such that for each $j=1, \ldots, n$ the function $G_{\alpha_{j}}$ can be written as

$$
G_{\alpha_{j}}=\mu_{\alpha_{j}} * P_{y_{j}}+c_{\alpha_{j}} y_{j} .
$$

Thus

$$
\begin{aligned}
u= & \sum_{|\alpha| \leq k} \partial^{\alpha_{1}}\left(\mu_{\alpha_{1}} * P_{y_{1}}+c_{\alpha_{1}} y_{1}\right) \ldots \partial^{\alpha_{n}}\left(\mu_{\alpha_{n}} * P_{y_{n}}+c_{\alpha_{n}} y_{n}\right) \\
= & \left(\mu_{\alpha_{1}} * P_{y_{1}}+c_{\alpha_{1}} y_{1}\right) \ldots\left(\mu_{\alpha_{n}} * P_{y_{n}}+c_{\alpha_{n}} y_{n}\right) \\
& +\sum_{0<|\alpha| \leq k}\left[\partial^{\alpha_{1}} \mu_{\alpha_{1}} \otimes \ldots \otimes \partial^{\alpha_{n}} \mu_{\alpha_{n}}\right] * \mathcal{P}_{(y)} \\
= & \left(\sum_{|\alpha| \leq k} \partial^{\alpha_{1}} \mu_{\alpha_{1}} \otimes \ldots \otimes \partial^{\alpha_{n}} \mu_{\alpha_{n}}\right) * \mathcal{P}_{(y)} \\
& +\sum c_{\alpha_{i_{1}}} y_{i_{1}} \ldots c_{\alpha_{i_{k}}} y_{i_{k}}\left(\mu_{\alpha_{j_{1}}} * P_{y_{j_{1}}}\right) \ldots\left(\mu_{\alpha_{j_{n-k}}} * P_{y_{j_{n-k}}}\right) \\
= & \left(\sum_{|\alpha| \leq k} \partial^{\alpha_{1}} \mu_{\alpha_{1}} \otimes \ldots \otimes \partial^{\alpha_{n}} \mu_{\alpha_{n}}\right) * \mathcal{P}_{(y)} \\
& +\sum c_{\alpha_{i_{1}}} y_{i_{1}} \ldots c_{\alpha_{i_{k}}} y_{i_{k}}\left(\left(\mu_{\alpha_{j_{1}}} \otimes \ldots \otimes \mu_{\alpha_{j_{n-k}}}\right) * \mathcal{P}_{\left(y_{j_{1}}, \ldots, y_{j_{n-k}}\right)}\right)
\end{aligned}
$$

where the sum collects all the possible cross-products containing at least one factor of the form $c_{\alpha_{i_{s}}} y_{i_{s}}$, without repetition.

We set

$$
T=\sum_{|\alpha| \leq k} \partial^{\alpha_{1}} \mu_{\alpha_{1}} \otimes \ldots \otimes \partial^{\alpha_{n}} \mu_{\alpha_{n}}
$$

and

$$
T_{j_{1}, \ldots, j_{n-k}}=\mu_{\alpha_{j_{1}}} \otimes \ldots \otimes \mu_{\alpha_{j_{n-k}}} .
$$

Using Lemma 9 and the fact that

$$
\int_{-\infty}^{\infty} w_{j}^{-2} d\left|\mu_{\alpha_{j}}\right|<\infty
$$

for each $j=1, \ldots, n$, we can readily see that $T \in w_{1}^{2} \mathcal{D}_{L^{1}}^{\prime} \otimes \ldots \otimes w_{n}^{2} \mathcal{D}_{L^{1}}^{\prime}$ and $T_{j_{1} \ldots j_{n-k}} \in w_{j_{1}}^{2} \mathcal{D}_{L^{1}}^{\prime} \otimes \ldots \otimes w_{j_{n-k}}^{2} \mathcal{D}_{L^{1}}^{\prime}$. Thus, the function $u$ can be represented as in (17) and the proof of 1) $\Longrightarrow 2$ ) is complete.

Conversely, let us prove that 2) implies 1). We assume that $u$ can be written as in (17) and we fix a term in (17), say

$$
y_{i_{1}} \ldots y_{i_{k}}\left(T_{j_{1}, \ldots, j_{n-k}} * \mathcal{P}_{\left(y_{j_{1}}, \ldots, y_{j_{n-k}}\right)}\right) .
$$


According to the one-dimensional version of Lemma 8, the distribution $T_{j_{1}, \ldots, j_{n-k}}$ can be written as a finite sum of terms of the form $\partial^{\alpha_{1}} f_{j_{1}} \otimes$ $\ldots \otimes \partial^{\alpha_{n-k}} f_{j_{n-k}}$ with $f_{j_{l}} \in L^{1}\left(w_{j_{l}}^{-2}\right)$. Then the representation stated in 1) follows for $y_{i_{1}} \ldots y_{i_{k}} \partial^{\alpha_{1}} f_{j_{1}} \otimes \ldots \otimes \partial^{\alpha_{n-k}} f_{j_{n-k}}$ by letting $G_{i}\left(z_{i}\right)=f_{i} * \mathcal{P}_{y_{i}}\left(x_{i}\right)$, for $i \in I$ and $G_{j}\left(z_{j}\right)=y_{j}$, for $j \in\{1,2, \ldots, n\} \backslash I$, where $I$ is defined as before.

This concludes the proof of Theorem 13 .

Remark 15. P. Sjögren observes in [14] that the quantities $\int_{\mathbb{R}^{n}} w^{-n-1}(x) d$ $\times|\mu|(x)+|a|$ and $\left\|W^{-n-1} y^{-1} u\right\|_{1, \infty}$ in Theorem 14 are equivalent, that is to say they are either both zero or their ratio remains bounded from below and from above by positive constants. Using this observation, it can be proved as in the Euclidean case ([2]) that there is a common bound on the number of terms, the order of the derivatives and the quasi-norms $\left\|W_{i}^{-2} y_{i}^{-1} G_{j}\right\|_{1, \infty}$ in the representation given in 1), if and only if the distribution $T_{j_{i}}$ belongs to a bounded set in $w_{j_{l}}^{2} \mathcal{D}_{L^{1}}^{\prime}(\mathbb{R})$ for each $j_{l}$, and $c_{I}$ belongs to a bounded subset of $\mathbb{C}$. These common bounds and bounded sets can be described by constants that are equivalent quantities.

\section{5. $\mathcal{S}^{\prime}$-convolvability with Poisson like kernels.}

As the results that follow show, in order to establish $\mathcal{S}^{\prime}$-convolvability, it is not as important to assume an exact formula for a kernel as it is to assume that the kernel satisfies the appropriate estimates. We will illustrate this heuristic principle in both the Euclidean case and the product domain case.

We begin with the Euclidean case, where we consider a kernel of the form $\phi_{y}(x)=\frac{1}{y^{n}} \phi\left(\frac{x}{y}\right), y>0$, for a function $\phi \in C^{\infty}\left(\mathbb{R}^{n}\right)$ fixed. We assume that $\phi$ is a non negative and integrable function such that $\int_{\mathbb{R}^{n}} \phi>0$. Moreover, we assume that $\phi$ satisfies the following estimates:

(a) There exists $\mu>n$ such that for every multi-index $\alpha$

$$
\left|\partial^{\alpha} \phi(x)\right| \leq C_{\alpha}(1+|x|)^{-(\mu+|\alpha|)}
$$

for some constant $C_{\alpha}=C_{\alpha, \mu, \phi}>0$.

(b) There exists a neighborhood $V$ of 0 in $\mathbb{R}^{n}$ such that for every $x \notin V$

$$
\phi(x) \geq C(1+|x|)^{-\mu}
$$

for some constant $C=C_{\mu, \phi}>0$. 
We shall denote by $\mathcal{G}_{\mu}$ the class of functions $\phi$ satisfying the properties described above.

It is clear that the classical Poisson kernel $P_{y}$ belongs to $\mathcal{G}_{n+1}$. Moreover, we have the following result, which turns out to be crucial in the formulation of necessary and sufficient conditions for the existence of the $\mathcal{S}^{\prime}$-convolution with the one-parameter family $\left\{\phi_{y}\right\}_{y>0}$, for $\phi \in \mathcal{G}_{\mu}$ fixed.

Proposition 16. Let $\phi \in \mathcal{G}_{\mu}$ fixed. Then the following statements hold.

(1) For each $\beta \in \mathcal{S}$ fixed, the function $\phi_{y} * \beta \in \dot{B}$. Furthermore, there exists a constant $C=C_{\mu, \phi, \beta}>0$ such that for every multi-index $\alpha$

$$
\left|\partial^{\alpha}\left(\phi_{y} * \beta\right)(x)\right| \leq \frac{C}{y^{n}}\left(1+\frac{|x|}{y}\right)^{-\mu}\left[\left\|\partial^{\alpha} \beta\right\|_{1}+\frac{1}{y^{\mu}}\left\||t|^{\mu} \partial^{\alpha} \beta\right\|_{1}\right] .
$$

(2) There exists an open ball $B_{\delta}(0)$ centered at zero with radius $\delta>0$, such that for every non-negative function $\beta \in C_{0}^{\infty}$ with $\operatorname{supp}(\beta) \subset$ $B_{\delta}(0)$, we have

$$
\left(\phi_{y} * \beta\right)(x) \geq C_{\mu} \frac{y^{\mu-n}}{(y+|x|)^{\mu}}\|\beta\|_{1}
$$

for a constant $C=C_{\mu, \phi, \beta}>0,|x|>2 \delta, 0<y \leq 1$.

Proof. Using (19), the proof of (21) follows the proof of Proposition 7 in $[1]$.

Concerning (22), since $\phi$ satisfies (20), there exists $\varepsilon>0$ such that

$$
\phi(x) \geq C(1+|x|)^{-\mu}
$$

for $C=C_{\mu, \phi, \varepsilon}>0$ and $|x| \geq \varepsilon$. Consider now a function $\beta \in C_{0}^{\infty}$ so that $\beta \geq 0, \beta=0$ if $|x| \geq \varepsilon$ and $\beta>0$ for $|x|<\varepsilon$.

If $|x|>2 \varepsilon,|t|<\varepsilon$ and $0<y \leq 1$, we have

$$
\frac{|x-t|}{y} \geq \frac{|x|}{y}-\frac{|t|}{y} \geq \frac{\varepsilon}{y} \geq \varepsilon
$$

and

$$
\begin{aligned}
\left(1+\frac{|x-t|}{y}\right)^{\mu} & \leq\left(1+\frac{|x|+\varepsilon}{y}\right)^{\mu} \\
& \leq C_{\mu}\left(1+\frac{|x|}{y}\right)^{\mu} \\
& =\frac{C_{\mu}}{y^{\mu}}(y+|x|)^{\mu} .
\end{aligned}
$$


Using these estimates we can obtain, for $|x|>2 \varepsilon$ and $0<y \leq 1$,

$$
\begin{aligned}
\left(\phi_{y} * \beta\right)(x) & =\int_{|t|<\varepsilon} \phi_{y}(x-t) \beta(t) d t \\
& \geq \frac{C_{\mu}}{y^{n}} \int_{|t|<\varepsilon}\left(1+\frac{|x-t|}{y}\right)^{-\mu} \beta(t) d t \\
& \geq \frac{C_{\mu}}{y^{n}} \frac{y^{\mu}}{(y+|x|)^{\mu}} \int_{|t|<\varepsilon} \beta(t) d t \\
& =C_{\mu}\|\beta\|_{1} \frac{y^{\mu-n}}{(y+|x|)^{\mu}} .
\end{aligned}
$$

This concludes the proof of Proposition 16.

Theorem 17. Let $T \in \mathcal{S}^{\prime}$ and $\mu>n$. Then the following statements are equivalent:

(1) $T \in w^{\mu} \mathcal{D}_{L^{1}}^{\prime}$.

(2) $T$ is $\mathcal{S}^{\prime}$-convolvable with $\phi_{y}$ for each $y>0$ and every $\phi \in \mathcal{G}_{\mu}$.

Proof. To show that 1) implies 2), we need to prove that $T\left(\phi_{y} * \beta\right)$ belongs to $\mathcal{D}_{L^{1}}^{\prime}$ for each $y>0$ and every $\phi \in \mathcal{G}_{\mu}$, if $T \in w^{\mu} \mathcal{D}_{L^{1}}^{\prime}$ and $\beta \in \mathcal{S}$.

We can use (21) to show that for each $y>0$ and each $\phi \in \mathcal{G}_{\mu}$ fixed, the function $(1+|x|)^{\mu}\left(\phi_{y} * \beta\right)$ belongs to $B$. Thus,

$$
T\left(\phi_{y} * \beta\right)=(1+|x|)^{-\mu}(1+|x|)^{\mu}\left(\phi_{y} * \beta\right)
$$

belongs to $\mathcal{D}_{L^{1}}^{\prime}$ for every $\beta \in \mathcal{S}$.

Conversely, if we assume that 2) holds, in order to conclude that $T \in$ $w^{\mu} \mathcal{D}_{L^{1}}^{\prime}$ it suffices to show that $T$ can be written as

$$
T=T_{1}+|x|^{\mu} T_{2},
$$

where $T_{1} \in \mathcal{E}^{\prime}, T_{2} \in \mathcal{D}_{L^{1}}^{\prime}$ and $T_{2}$ is zero in a neighborhood of zero ([2]). That this is the case follows quite readily from (22).

This concludes the proof of Theorem 17 .

In the product domain case we can consider as a Poisson like kernel the function $\phi_{1} \otimes \ldots \otimes \phi_{n}$, where $\phi_{j}\left(x_{j}\right)=\phi\left(x_{j}\right), j=1, \ldots, n, \phi \in \mathcal{G}_{\mu}(\mathbb{R})$, $\mu>1$. For $(y)=\left(y_{1}, \ldots, y_{n}\right)>(0)$, let us denote

$$
\Phi_{(y)}(x)=\prod_{J=1}^{n} \phi_{y_{j}}\left(x_{j}\right) .
$$


The product domain Poisson kernel $\mathcal{P}_{(y)}$ is a particular case of $\Phi_{(y)}$. Using the one-dimensional version of Proposition 16, we can prove the following result.

Theorem 18. Let $T \in \mathcal{S}^{\prime}$ and $\mu>1$. Then the following statements are equivalent:

(i) $T \in w_{1}^{\mu} \ldots w_{n}^{\mu} \mathcal{D}_{L^{1}}^{\prime}$.

(ii) $T$ is $\mathcal{S}^{\prime}$-convolvable with $\Phi_{(y)}$ for each $(y)>(0)$ and every $\phi \in \mathcal{G}_{\mu}(\mathbb{R})$.

\section{References}

[1] J. Alvarez, M. Guzmán-Partida and U. Skórnik, $\mathcal{S}^{\prime}$-convolvability with the Poisson kernel in the Euclidean case and the product domain case, Studia Math., 156 (2003), 143-163.

[2] J. Alvarez, M. Guzmán-Partida and S. Pérez-Esteva, Harmonic extensions of distributions, Math. Nachr., to appear.

[3] J. Barros-Neto, An Introduction to the Theory of Distributions, Marcel Dekker, 1973.

[4] H. Bremermann, Distributions, Complex Variables and Fourier Transform, Addison-Wesley, 1965.

[5] P. Dierolf and J. Voigt, Convolution and $\mathcal{S}^{\prime}$-convolution of distributions, Collect. Math., 29 (1978), 185-196.

[6] T. M. Flett, On the rate of growth of mean values of holomorphic and harmonic functions, Proc. London Math. Soc., 20 (3) (1970), 749-768.

[7] Y. Hirata and H. Ogata, On the exchange formula for distributions, J. Sci. Hiroshima Univ., Ser. A, 22 (1958), 147-152.

[8] J. Horváth, Composition of hypersingular integral operators, Applicable Analysis, 7 (1978), 171-190.

[9] J. Horváth, Convolution de noyaux hypersinguliers, Séminaire Initiation à l'Analyse, (G. Choquet, M. Rogalski and J. SaintRaymond) (1979/1980), 8, January 1980, 1-17.

[10] N. Ortner, Sur quelques proprietés des espaces $\mathcal{D}_{L^{p}}^{\prime}$ de Laurent Schwartz, Boll. Un. Mat. Ital. B, 2 (6) (1) (1983), 353-375.

[11] N. Ortner and P. Wagner, Applications of weighted $\mathcal{D}_{L^{p}}^{\prime}$-spaces to the convolution of distributions, Bull. Polish Acad. Sci. Math., 37 (1990), 579-595.

[12] L. Schwartz, Théorie des Distributions, Hermann, 1966. 
[13] R. Shiraishi, On the definition of convolutions for distributions, J. Sci. Hiroshima Univ., Ser. A, 23 (1959), 19-32.

[14] P. Sjögren, Characterizations of Poisson integrals and $H^{p}$ spaces, Report 5 (1975), Department of Mathematics, Chalmers University of Technology and The University of Göteborg, published as Weak $L^{1}$ characterizations of Poisson integrals, Green potentials, and $H^{p}$ spaces, Trans. Amer. Math. Soc., 233 (1977), 179-196.

[15] E. M. Stein and G. Weiss, Fourier Analysis on Euclidean Spaces, Princeton University Press, 1971.

Department of Mathematics

New Mexico State University

Las Cruces, NM 88003

USA

(E-mail : jalvarez@nmsu.edu)

Departamento de Matemáticas

Universidad de Sonora

Hermosillo, Sonora 83000

México

(E-mail : martha@gauss.mat.uson.mx)

Instituto de Matemáticas, Unidad Cuernavaca

Universidad Nacional Autónoma de México

Cuernavaca, Morelos 62251

México

(E-mail :salvador@matcuer.unam.mx) 


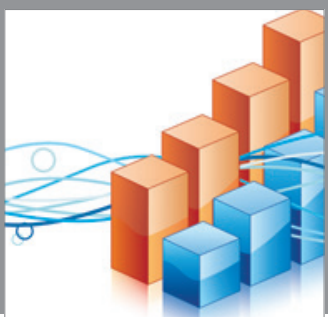

Advances in

Operations Research

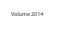

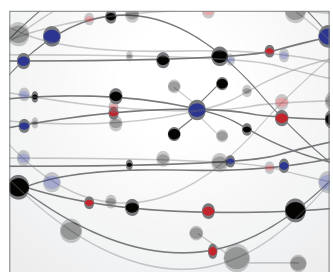

\section{The Scientific} World Journal
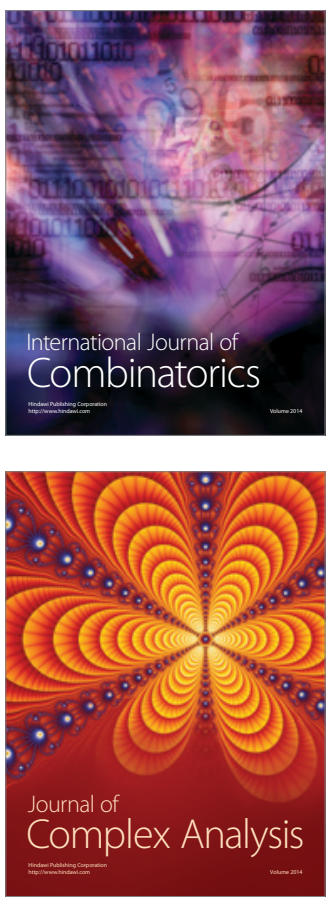

International Journal of

Mathematics and

Mathematical

Sciences
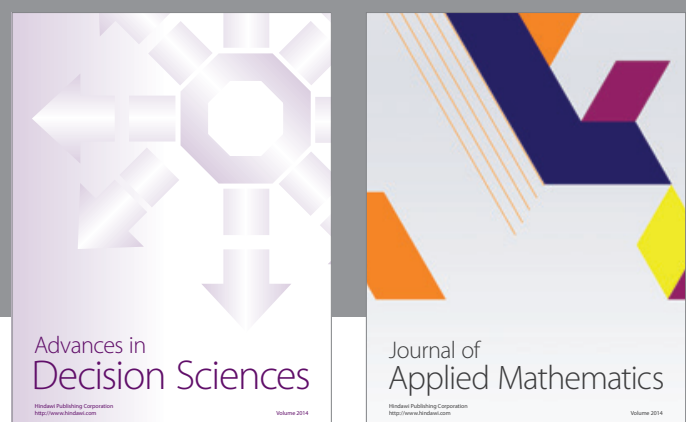

Journal of

Applied Mathematics
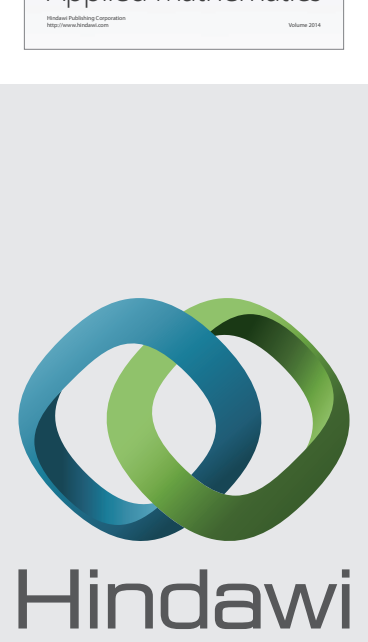

Submit your manuscripts at http://www.hindawi.com
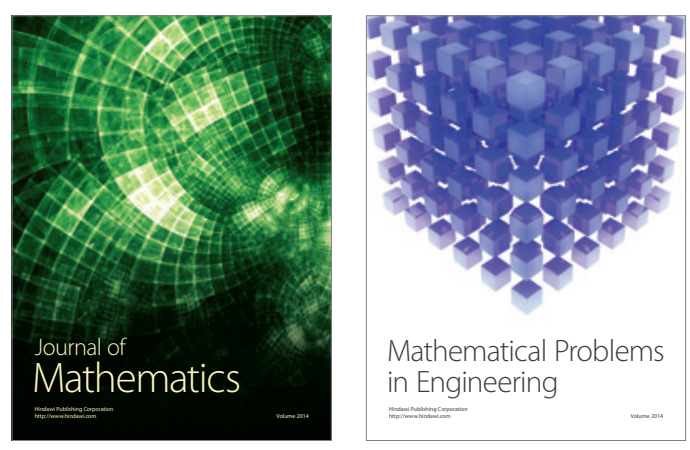

Mathematical Problems in Engineering
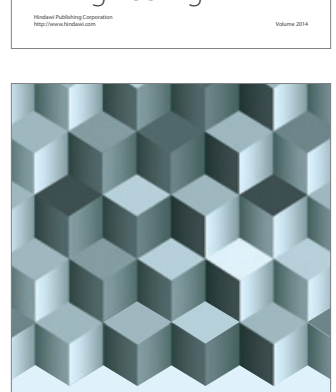

Journal of

Function Spaces
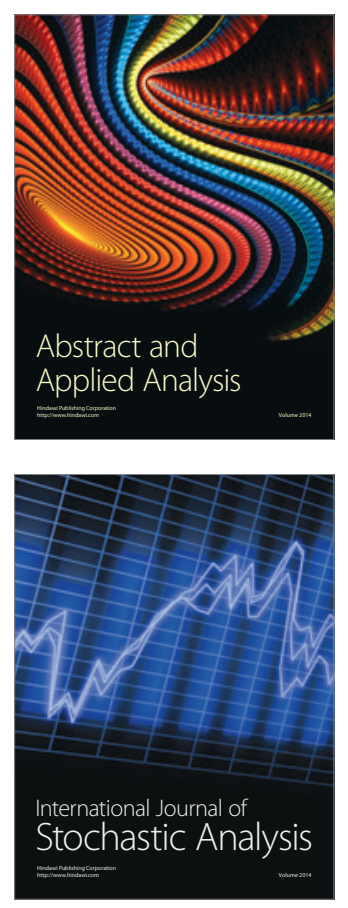

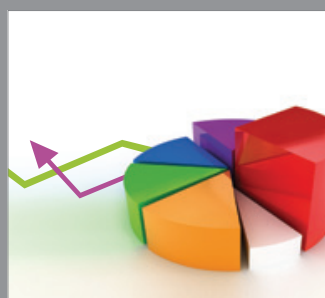

ournal of

Probability and Statistics

Promensencen
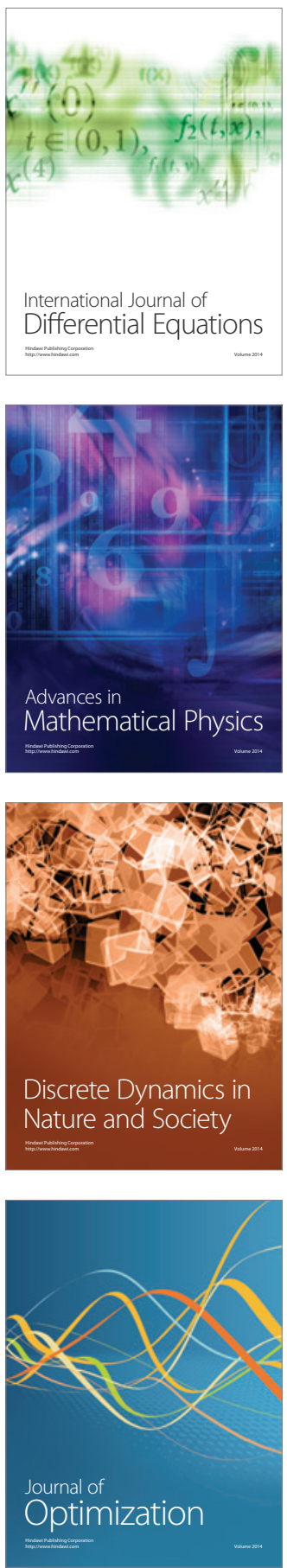